\title{
CHARACTERIZATION OF CLOSED IDEALS WITH BOUNDED APPROXIMATE IDENTITIES IN COMMUTATIVE BANACH ALGEBRAS, COMPLEMENTED SUBSPACES OF THE GROUP VON NEUMANN ALGEBRAS AND APPLICATIONS
}

\author{
ANTHONY TO-MING LAU AND ALI ÜLGER
}

\begin{abstract}
Let $A$ be a commutative Banach algebra with a BAI (=bounded approximate identity). We equip $A^{* *}$ with the (first) Arens multiplication. To each idempotent element $u$ of $A^{* *}$ we associate the closed ideal $I_{u}=\{a \in A$ : $a u=0\}$ in $A$. In this paper we present a characterization of the closed ideals of $A$ with BAI's in terms of idempotent elements of $A^{* *}$. The main results are: a) A closed ideal $I$ of $A$ has a BAI iff there is an idempotent $u \in A^{* *}$ such that $I=I_{u}$ and the subalgebra $A u$ is norm closed in $A^{* *}$. b) For any closed ideal $I$ of $A$ with a BAI, the quotient algebra $A / I$ is isomorphic to a subalgebra of $A^{* *}$. We also show that a weak* closed invariant subspace $X$ of the group von Neumann algebra $V N(G)$ of an amenable group $G$ is naturally complemented in $V N(G)$ iff the spectrum of $X$ belongs to the closed coset ring $\Re_{c}\left(G_{d}\right)$ of $G_{d}$, the discrete version of $G$. This paper contains several applications of these results.
\end{abstract}

\section{INTRODUCTION}

Let $A$ be a commutative Banach algebra with a BAI. We equip the second dual $A^{* *}$ of $A$ with the first Arens multiplication, the construction of which will be recalled in the next section. In this paper we present a characterization of the closed ideals of $A$ possessing a BAI in terms of idempotent elements of the algebra $A^{* *}$ and consider some applications of the main results. To explain the content of the paper and the background results we need some terminology and notation.

For $f \in A^{*}$ and $a \in A$, by $a . f$ we denote the functional on $A$ defined by $\langle a . f, b\rangle=$ $\langle f, a b\rangle$. By a "projection" we mean a bounded linear projection. A projection $P: A^{*} \rightarrow A^{*}$ is said to be "invariant" (or $A$-invariant) if, for all $a \in A$ and $f \in A^{*}$, the equality $P(a . f)=a . P(f)$ holds. Similarly, a closed subspace $X$ of $A^{*}$ is said to be "invariant" if, for each $a \in A$ and $f \in X$, the functional a. $f$ is in $X$ (i.e. $X$ is an $A$-module for the natural action $(a, f) \mapsto a . f)$. If there is an invariant projection from $A^{*}$ onto a closed invariant subspace $X$ of $A^{*}$, then $X$ is said to be "invariantly complemented in $A^{*}$ ". For $a \in A$ and $u \in A^{* *}$, by $a u$ we denote the (Arens) product of $a$ and $u$ in $A^{* *}$. For $f \in A^{*}$ and $u \in A^{* *}$, by $u$. $f$ we denote the functional on $A$ defined by $\langle a, u . f\rangle=\langle a u, f\rangle$. For a locally compact group $G$, by $\Re\left(G_{d}\right)$ we denote the Boolean ring generated by the cosets of the subgroups of $G_{d}$,

Received by the editors August 17, 2012.

2010 Mathematics Subject Classification. Primary 46H20, 43A25, 43A46; Secondary 43A22.

Key words and phrases. Commutative Banach algebras, invariant complementation, idempotent, Fourier algebra, Fourier-Stieltjes algebra, group von Neumann algebra, closed ideal, coset ring, bounded approximate identity, multiplier, Arens product.

The first author was supported by NSERC grant MS 100. 
the group $G$ with the discrete topology. By $\Re_{c}\left(G_{d}\right)$ we denote the subring of $\Re\left(G_{d}\right)$ consisting of the closed elements of the ring $\Re\left(G_{d}\right)$. Finally, by $\Delta(A)$ we denote the Gelfand spectrum of $A$.

As stated above, our main aim in this paper is to characterize the closed ideals of the algebra $A$ with BAI in terms of the idempotent elements of $A^{* *}$. The existence of a BAI in a closed ideal $I$ is equivalent to the existence of an invariant projection from $A^{*}$ onto the annihilator $I^{\perp}$ of $I$ in $A^{*}$ ([10, p. 245, Theorem 1] and [5, Proposition 6.4]). A natural problem that arises in this context is this: If there is a projection from $A^{*}$ onto $I^{\perp}$, when is there an invariant projection from $A^{*}$ onto $I^{\perp}$ ? These two problems (the existence of a BAI in a closed ideal $I$ and the existence of an invariant projection from $A^{*}$ onto $I^{\perp}$ ) have been studied by a considerable number of mathematicians in the settings of the group algebra $L^{1}(G)$ of a locally compact abelian group $G$ and also Eymard's Fourier algebra $A(G)$ of a locally compact amenable group $G$ [4. (See below for a short history of these problems.) In this paper we study these two problems from an abstract point of view with special emphasis on the Fourier algebra $A(G)$.

If a closed ideal $I$ of the algebra $A$ has a BAI, then there is an idempotent element $u$ in $A^{* *}$ such that $I=I_{u}$, where $I_{u}=\{a \in A: a u=0\}$. However, not every ideal of the form $I_{u}$ has a BAI. The first and main problem that we study in this paper is:

Problem (A). Characterize the idempotent elements $u$ of $A^{* *}$ for which the closed ideal $I_{u}=\{a \in A: a u=0\}$ has a BAI.

It has turned out that a closed ideal $I$ of $A$ has a BAI iff there is an idempotent element $u \in A^{* *}$ such that $I_{u}=I$ and the space $A u=\{a u: a \in A\}$, which is a subalgebra of $A^{* *}$, is norm closed in the algebra $A^{* *}$. In this case, the Banach algebras $A / I_{u}$ and $A u$ are isomorphic so that the Banach space $A / I_{u}$ possesses all the hereditary properties of the Banach space $A^{* *}$. For instance, if the space $A^{* *}$ is weakly sequentially complete, then so is the quotient space $A / I_{u}$. We recall that, among many other Banach algebras, the second dual of the group algebra $L^{1}(G)$ and that of the Fourier algebra $A(G)$ of any locally compact group $G$ are weakly sequentially complete. More generally, the predual of any von Neumann algebra is weakly sequentially complete. The subalgebra $A u$ is apparently more transparent than the quotient algebra $A / I_{u}$. The fact that the algebra $A / I_{u}$ is isomorphic to the algebra $A u$ may help to solve certain problems about the quotient algebra $A / I_{u}$, such as the determination of the multiplier algebra of $A / I_{u}$, for instance.

As mentioned above, Problem (A) is equivalent to another much studied problem. This is

Problem (B). When is a complemented weak* closed invariant subspace of $A^{*}$ invariantly complemented in $A^{*}$ ?

The second problem we study in this paper is very closely related to this version of Problem (A). Here the problem is to put on the projection $P: A^{*} \rightarrow I^{\perp}$ a condition as weak as is possible but nevertheless sufficient to guarantee the existence of a BAI in the ideal $I$. The most natural condition to put on a projection $P: A^{*} \rightarrow I^{\perp}$ seems to be, for each $\gamma \in \Delta(A)$, either $P(\gamma)=\gamma$ or $P(\gamma)=0$. Any invariant projection $P: A^{*} \rightarrow I^{\perp}$ satisfies this condition. In Section 3 we show that in some important cases, including the Fourier algebra $A(G)$ of an amenable group $G$, this condition is both necessary and sufficient for the ideal $I$ to have a BAI. 
The full story of these two problems (A and B) is too long to state here; these problems have been studied by a considerable number of mathematicians since the 1960s. Here we mention only the most relevant works on the subject matter of this paper. In 34 Rudin proved that every complemented closed ideal of the group algebra $L^{1}(G)$ of a compact group $G$ is invariantly complemented and has a BAI. In his celebrated memoir 32 Rosenthal considered the dual version of this problem and proved, among other results, that for any locally compact abelian group $G$, the spectrum of a weak* closed translation invariant complemented subspace $X$ of $L^{\infty}(G)$ is in the closed coset ring $\Re_{c}\left(\widehat{G}_{d}\right)$ and $X$ is complemented by a projection that commutes with the translations by the elements of $G$. In [14, Theorem A] Gilbert, completing the work of Rosenthal, showed that any weak* closed invariant subspace of $L^{\infty}(G)$ whose spectrum is in the ring $\Re_{c}\left(\widehat{G}_{d}\right)$ is invariantly complemented so that any complemented weak* closed invariant subspace of $L^{\infty}(G)$ is invariantly complemented. This latter result is valid, more generally, for any amenable Banach algebra [2, Corollary 2.9.59]. The corresponding results for the Fourier algebra $A(G)$ of an amenable locally compact group $G$ (we recall here that the algebra $A(G)$ is amenable only if $G$ has an abelian subgroup of finite index [8]) have been studied by several authors $([24$, [26, [5], [6] and [39], among others), but the results are more involved and are not as complete as in the $L^{1}(G)$ case. The closed ideals with BAI of the algebra $L^{1}(G)(G$ is abelian) and those of the Fourier algebra $A(G)$ ( $G$ is amenable) of a locally compact group $G$ are characterized in the papers 30] and 9, respectively. These are exactly the closed ideals of the form $k(E)=\left\{a \in L^{1}(G): \widehat{a}(E)=\{0\}\right\}$ for $E \in \Re_{c}\left(\widehat{G}_{d}\right)$ and $k(E)=\{a \in A(G): a(E)=\{0\}\}$ for $E \in \Re_{c}\left(G_{d}\right)$, respectively.

In this paper we study exactly the same problems (A and B) for a general commutative Banach algebra $A$ with a BAI. Since in this case there is no functional on $A^{*}$ such as an "invariant mean" permitting the use of the averaging techniques and since there is no structure such as the coset ring $\Re\left(G_{d}\right)$ or Bohr compactification on the Gelfand spectrum of the algebra $A$ to reduce the problem to known cases, the only way of characterizing the closed ideals of $A$ with BAI that appeared possible to us is the one in terms of the idempotent elements of $A^{* *}$. This is the approach we have followed. This approach is essentially functional analytic and has its own advantages, the main one being the realization of the quotient algebra $A / I_{u}$ as a closed subalgebra, namely $A u=\{a u: a \in A\}$, of $A^{* *}$. We present several applications of this result. Concerning the second aspect of the main problem, we show that there is another class of projections $P: A^{*} \rightarrow A^{*}$, closely related to the invariant projections, that also plays an important role in the study of Problem (B). We call these projections "natural projections". These are the projections $P: A^{*} \rightarrow A^{*}$ such that, for each $\gamma \in \Delta(A)$, either $P(\gamma)=\gamma$ or $P(\gamma)=0$. As mentioned above, a closed ideal $I$ of the Fourier algebra $A=A(G)$ of an amenable group $G$ has a BAI iff $I^{\perp}$ is "naturally complemented" in $A(G)^{*}$.

In Section 1 we have collected a few preliminary results about Arens multiplications, the Fourier and the Fourier-Stieltjes algebras $A(G)$ and $B(G)$, and the coset ring $\Re(G)$. The main results are in Sections 2 and 3 (Theorem 2.7, Corollary 2.8 and Theorem 3.3). The applications are in Section 4 (Theorem 4.1, Corollary 4.3 and Proposition 4.5). The main ingredients of the proofs are the basic results of functional analysis and Banach algebras. 
Finally, we would like to emphasize here that most of the results presented in this paper (especially the results in Sections 2 and 4) are valid for noncommutative Banach algebras as well. We have chosen to work with commutative Banach algebras because, historically, the most studied cases, the group algebra $L^{1}(G)(G$ abelian) and the Fourier algebra $A(G)$ ( $G$ amenable), are commutative, as are our main examples.

\section{Notation AND PRELIMINARY RESUlts}

Our notation and terminology are standard, and some of them have already been introduced in the preceding section. In addition to these, we need a few facts related to the Arens multiplications, Fourier algebra $A(G)$ and the coset ring $\Re(G)$.

Let $A$ be a Banach algebra. We always regard $A$ as canonically embedded into its second dual $A^{* *}$. By $\langle a, f\rangle$ (and also by $\langle f, a\rangle$ ) we denote the natural duality between $A$ and $A^{*}$, and by $A_{1}$ the closed unit ball of $A$. On $A^{* *}$ there are two Banach algebra multiplications extending that of $A$, under each of which $A^{* *}$ is a Banach algebra. These multiplications are known as the first and second Arens multiplications of $A^{* *}$. In this paper we shall use only the first of them, the construction of which we recall now. This multiplication is constructed in three steps as follows. For $a, b$ in $A, f$ in $A^{*}$ and $n, m$ in $A^{* *}$, the elements $a . f, n . f$ of $A^{*}$, and $m n$ of $A^{* *}$ are defined, respectively, as follows:

$$
\begin{aligned}
\langle a . f, b\rangle & =\langle f, b a\rangle, \\
\langle n . f, a\rangle & =\langle n, a . f\rangle
\end{aligned}
$$

and

$$
\langle m n, f\rangle=\langle m, n . f\rangle .
$$

For $m \in A^{* *}$ fixed, the multiplication operator $n \mapsto n m$ is weak* - weak* continuous on $A^{* *}$ but, for $n$ fixed, the multiplication operator $m \mapsto n m$ is in general not weak* - weak* continuous on $A^{* *}$. Even when the algebra $A$ is commutative, under this multiplication $A^{* *}$ is in general not commutative. However, if the algebra $A$ is commutative, for each $m \in A^{* *}$ and $a \in A$, we have $a m=m a$. We also recall that, if $\left(a_{i}\right)_{i \in I}$ and $\left(b_{j}\right)_{j \in J}$ are two bounded nets in $A$ converging, respectively, to $n$ and $m$ in the weak* topology of $A^{* *}$, then the iterated weak*-limits $\lim _{i} \lim _{j} a_{i} b_{j}$ exist and

$$
m n=\lim _{i} \lim _{j} a_{i} b_{j} .
$$

For instance, if $I$ is a closed ideal of $A$, then this iterated limit definition of Arens multiplication shows that $I^{* *}$ is also a closed ideal in $A^{* *}$. This is a fact that we shall use without reference.

Now let $\left(e_{i}\right)_{i \in I}$ be a bounded approximate identity (=BAI) in the algebra $A$. Then any weak ${ }^{*}$ cluster point $e$ of the net $\left(e_{i}\right)_{i \in I}$ in $A^{* *}$ is a right identity in $A^{* *}$ (i.e. $m e=m$, for all $m \in A^{* *}$ ). Conversely, any right identity of $A^{* *}$ is a weak* cluster point of some BAI in $A$ [2, Proposition 2.9.16]. For each right identity $e$ of $A^{* *}$ and for each $a \in A$, the equalities $e a=a e=a$ hold. Such a right identity $e$ will play an important role in the proofs of the results presented in this paper. We denote by $A A^{*}$ the space of the functionals of the form a.f $\left(a \in A\right.$ and $\left.f \in A^{*}\right)$. Since the algebra $A$ has a BAI, the space $A A^{*}=\left\{a . f: a \in A\right.$ and $\left.f \in A^{*}\right\}$ 
is a closed subspace of $A^{*}[11,32.22]$. We also note that each $\gamma \in \Delta(A)$ is also multiplicative on $A^{* *}$, as one can easily see. The reader can find ample information on the Arens multiplications, BAI, amenability and related notions in the books 2], 10] and 37.

Now suppose that the algebra $A$ is commutative, semisimple and regular. For $a \in A$, by $\widehat{a}$ we denote the Gelfand transform of $a$. To each closed subset $F$ of $\Delta(A)$, we associate two ideals,

$$
\begin{aligned}
& k(F)=\{a \in A: a=0 \text { on } F\} \text { and } \\
& j(F)=\{a \in A: \text { The support of } \widehat{a} \text { is compact and disjoint from } F\} .
\end{aligned}
$$

The ideals $\overline{j(F)}$ and $k(F)$ are, respectively, the smallest and the largest closed ideals of $A$ with hull $F$. When these ideals are equal, the set $F$ is said to be a set of synthesis. On sets of synthesis the reader can find ample information in Chapter 5 of the book 21 .

Now let $G$ be a locally compact group equipped with its left Haar measure. In his seminal paper P. Eymard [4 has associated to the group $G$ two important commutative Banach algebras. These are the Fourier algebra $A(G)$ and the FourierStieltjes algebra $B(G)$ of $G$. The Fourier -Stieltjes algebra $B(G)$ is the linear span of the set of the continuous positive definite complex valued functions on $G$. This is also the space of the coefficient functions of the unitary representations of the group $G$. More precisely, given $u \in B(G)$, there exists a unitary representation $\pi$ of $G$ and two vectors $\xi$ and $\eta$ in the representation space $H(\pi)$ of $\pi$ such that, for $x \in G$,

$$
u(x)=\langle\pi(x) \xi, \eta\rangle .
$$

Equipped with the norm $\|u\|=\inf _{\xi, \eta}\|\xi\| .\|\eta\|$, where the infimum is taken on all $\xi$ and $\eta$ satisfying the preceding equality, and the pointwise multiplication, $B(G)$ is a commutative Banach algebra. The Banach algebra $B(G)$ is also the dual space of the group $C^{*}$-algebra $C^{*}(G)$. The Fourier algebra $A(G)$ is the closed ideal of $B(G)$ generated by the elements of $B(G)$ with compact support. The algebra $A(G)$ can also be defined as the set of the coordinate functions of the left regular representation of $G$ in $L^{2}(G)$. When $G$ is abelian, via the Fourier transform,

$$
A(G)=L^{1}(\widehat{G}), \quad B(G)=M(\widehat{G}) \text { and } C^{*}(G)=C_{0}(\widehat{G}) .
$$

Since their introduction in the 1960s by Eymard, these algebras have been constant objects of study and they are among the most important Banach algebras of harmonic analysis. Both of the Banach algebras $A(G)$ and $B(G)$ are semisimple. Moreover, the Fourier algebra $A(G)$ is regular and Tauberian. The Gelfand spectrum of $A(G)$ can be identified with the group $G$. Each element of $G$ acts on $A(G)$ as a point evaluation. The algebra $A(G)$ has a BAI iff the group $G$ is amenable 28. As is well known, the abelian groups and the compact groups are amenable. On the negative side, the noncompact semisimple Lie groups, as well as any group containing a closed free subgroup generated by more than one generator, are not amenable. The reader can find ample information on this notion in the books [18] and 31 .

By $\Re\left(G_{d}\right)$ we denote the Boolean ring generated by the cosets of the subgroups $G_{d}$, the group $G$ with the discrete topology. By $\Re_{c}\left(G_{d}\right)$ we denote the "closed coset ring of $G$ ". This is the ring consisting of the closed elements of the coset ring $\Re\left(G_{d}\right)$. For abelian groups, the structure of the elements of the ring $\Re_{c}\left(G_{d}\right)$ 
have been described by Gilbert [14] and Schreiber [38, independently. Forrest 7 ] verified that the same description is also valid in the nonabelian case.

Finally, for the convenience of the reader, we recall the following classical result that we will use repeatedly. This result can be found in Rudin's book 35] as Theorem 4.14 .

Theorem (A). Let $X, Y$ be two Banach spaces and $T: X \rightarrow Y$ a bounded linear operator. Then the following assertions are equivalent:

1) $T(X)$ is closed in $Y$.

2) $T^{*}\left(Y^{*}\right)$ is norm closed in $X^{*}$.

3) $T^{*}\left(Y^{*}\right)$ is weak $k^{*}$ closed in $X^{*}$.

\section{Characterizations of the Closed ideals with BAI}

Throughout this section $A$ will be a commutative Banach algebra with a BAI. The main results of this section are Theorem 2.7 and Corollary 2.8, where the two results announced in the abstract are stated and proved.

We always assume that $A^{* *}$ is equipped with the first Arens multiplication as defined in the preceding section. The letter $e$ will always denote a right identity in $A^{* *}$. Even if the algebra $A$ is semisimple and its Gelfand spectrum $\Delta(A)$ is connected, the algebra $A^{* *}$ has in general many idempotent elements, as this is the case, for instance, when $A$ is the $C^{*}$ - algebra $C_{0}(\Omega)$ of a connected locally compact space $\Omega$. Indeed, the characteristic function of each open subset of $\Omega$, considered as an element of $C_{0}(\Omega)^{* *}$, is an idempotent. To each idempotent element $u$ of $A^{* *}$, we associate the closed ideal

$$
I_{u}=\{a \in A: a u=0\} .
$$

The ideal $I_{u}$ need not have a BAI (see Remark $2.6 \mathrm{~d}$ ) below). In this section our aim is to find out when the ideal $I_{u}$ has a BAI. We start with some preliminary results.

The first lemma shows that every closed ideal $I$ of $A$ with a BAI is of the form $I_{u}$ for some idempotent elements $u$ of $A^{* *}$. For that reason we shall be concerned mostly with the ideals of the form $I_{u}$.

Lemma 2.1. Let $I$ be a closed ideal of $A$ with a BAI. Then $I=I_{u}$ for some idempotent element $u$ of $A^{* *}$.

Proof. Let $\left(d_{\alpha}\right)_{\alpha \in I}$ be a BAI in $I$ and $d$ be a weak* cluster point of the net $\left(d_{\alpha}\right)_{\alpha \in I}$ in $A^{* *}$. Then $d$ is an idempotent and a right identity in $I^{* *}$. Set $u=e-e d$. Then

$$
u^{2}=(e-e d)(e-e d)=e-e d-e d+e d e d=e-e d=u
$$

so that $u$ is an idempotent. Since $d$ is a right identity in $I^{* *}$, for each $a \in I, a d=a$. So, for $a \in I, a u=a(e-e d)=0$. Hence $I \subseteq I_{u}$. Conversely, if for some $a \in A$,

$$
a u=a(e-e d)=0,
$$

so that $a=a d$. As $d \in I^{* *}$ and $I^{* *}$ is a two-sided ideal in $A^{* *}$, the product $a d$ is in $I^{* *}$. Since $a=a d$ and $a \in A$, necessarily $a \in I$ so that $I=I_{u}$.

The next result is known ([10, p. 245, Theorem 1.20] and [5, Proposition 6.4]). We shall include a proof of this result since it plays an important role in this paper. This lemma shows that, as mentioned in the introduction, Problem (A) and Problem (B) are indeed equivalent. 
Lemma 2.2. Let $I$ be a closed ideal of $A$. The ideal $I$ has a BAI iff the weak* closed invariant subspace $I^{\perp}$ of $A^{*}$ is invariantly complemented in $A^{*}$.

Proof. Suppose first that $I$ has a BAI $\left(d_{\alpha}\right)_{\alpha \in I}$. With the notation of the preceding lemma, let $u=e-e d$. Then $I=I_{u}$. Define the linear operator $P: A^{*} \rightarrow A^{*}$ by $P(f)=u . f$. The operator $P$ is a projection and $P(a . f)=u a . f=a . P(f)$ for all $a \in A$ and $f \in A^{*}$ so that $P$ is invariant. For each $f \in A^{*}, u . f \in I^{\perp}$ since $I=I_{u}$ and $u . f=P(f)$. Hence $P\left(A^{*}\right) \subseteq I^{\perp}$. The reverse inclusion is also true. Indeed, since for all $a \in A, a d_{\alpha}$ is in $I$, for any $f \in I^{\perp}$, we have $\left\langle f, a d_{\alpha}\right\rangle=0$. Hence, for each $a \in A, a d \in I^{\perp \perp}$ and

$$
\langle a, u . f\rangle=\langle a(e-e d), f\rangle=\langle a, f\rangle
$$

so that $f=u . f=P(f)$. Hence $P\left(A^{*}\right)=I^{\perp}$.

Conversely, suppose that there is an invariant projection $P$ from $A^{*}$ onto $I^{\perp}$. Then

$$
I^{\perp \perp}=P\left(A^{*}\right)^{\perp}=\operatorname{Ker}\left(P^{*}\right)=\left(I-P^{*}\right)\left(A^{* *}\right)
$$

so that the element $v=e-P^{*}(e)$ is in the second dual $I^{* *}=I^{\perp \perp}$ of $I$. Also, since $P$ is invariant, for $a \in A, a P^{*}(e)=P^{*}(a)$. So, for $a \in I$ and $f \in A^{*}$, we have

$$
\left\langle P^{*}(a), f\right\rangle=\langle a, P(f)\rangle=0 .
$$

Hence, for $a \in I, a P^{*}(e)=P^{*}(a)=0$ so that $a v=a\left(e-P^{*}(e)\right)=a$. This implies that, for $m \in I^{* *}, m v=m$. This shows that $v$ is a right identity in $I^{* *}$ so that the ideal $I$ has a BAI.

For any idempotent element $u$ of $A^{*}$, we define the space $u . A^{*}$ as

$$
u . A^{*}=\left\{u . f: f \in A^{*}\right\} .
$$

Since the mapping $P: A^{*} \rightarrow A^{*}$, defined by $P(f)=u$.f, is a projection, u. $A^{*}$ is a norm closed invariant subspace of $A^{*}$ but is not necessarily weak* closed (see Remark 2.6 e) below). The space $u . A^{*}$ is included in the space $I_{u}^{\perp}$, but the equality $I_{u}^{\perp}=u . A^{*}$ does not hold unless the space $u . A^{*}$ is weak* closed in $A^{*}$.

The next lemma plays a key role in the proof of the main result of this section.

Lemma 2.3. A closed ideal $I$ of $A$ has a BAI iff there is an idempotent $u \in A^{* *}$ such that $I=I_{u}$ and the space $u . A^{*}$ is weak* closed in $A^{*}$. In this case the equality $I_{u}^{\perp}=u . A^{*}$ holds.

Proof. Suppose that $I$ has a BAI. Let $d$ be a right identity in $I^{* *}$. Then, as seen during the proof of Lemma 2.1, the element $u=e-e d$ of $A^{* *}$ is an idempotent and $I_{u}=I$. Let us see that the space $u . A^{*}$ is weak ${ }^{*}$ closed in $A^{*}$. To prove this it is enough to prove that $u . A^{*}=I_{u}^{\perp}$. Since $I_{u}^{\perp \perp}$ is an ideal in $A^{* *}$ and $d \in I_{u}^{\perp \perp}$, for each $a \in A$ the product $a d$ is in the ideal $I_{u}^{\perp \perp}$. Here, of course, we naturally identify $I^{\perp \perp}$ and $I^{* *}$. Hence, for each $a$ in $A$ and $f$ in $I_{u}^{\perp},\langle a d, f\rangle=0$. It follows that, for $f \in I_{u}^{\perp}$,

$$
\langle a, u . f\rangle=\langle a u, f\rangle=\langle a-a d, f\rangle=\langle a, f\rangle .
$$

Hence $f=u$.f so that $I_{u}^{\perp}=u . A^{*}$, the space $u . A^{*}$ is weak ${ }^{*}$ closed in $A^{*}$ and the equality $I_{u}^{\perp}=u \cdot A^{*}$ holds.

Conversely, suppose that for some idempotent $u \in A^{* *}, I=I_{u}$ and the space $u . A^{*}$ is weak* closed in $A^{*}$. Then, as the Hahn-Banach Theorem shows, $I_{u}^{\perp}=u . A^{*}$. The mapping $P: A^{*} \rightarrow A^{*}$, defined by $P(f)=u$. $f$, is an invariant projection from $A^{*}$ onto $I_{u}^{\perp}$ so that, by the preceding lemma, the ideal $I$ has a BAI. 
We consider the idempotent $u \in A^{* *}$ as a function on $\Delta(A)$. Since $u$ is an idempotent and since each $\gamma \in \Delta(A)$ is also multiplicative on $A^{* *}$, for each $\gamma \in$ $\Delta(A), u(\gamma)$ is either one or zero. Let

$$
E_{u}=\{\gamma \in \Delta(A): u(\gamma)=1\}
$$

Since $u . \gamma=u(\gamma) \gamma$, we have $u . A^{*} \cap \Delta(A)=E_{u}$. Hence, if the space $u . A^{*}$ is weak ${ }^{*}$ closed in $A^{*}$, the set $E_{u}$ is closed in $\Delta(A)$. We record this observation in the next corollary.

Corollary 2.4. Let $u \in A^{* *}$ be an idempotent. If the space $u . A^{*}$ is weak ${ }^{*}$ closed in $A^{*}$, then the set $E_{u}=\{\gamma \in \Delta(A): u(\gamma)=1\}$ is closed in $\Delta(A)$.

If $u . A^{*}$ is weak ${ }^{*}$ closed in $A^{*}$, then the set $E_{u}$ is the hull of the ideal $I_{u}$. If, in addition, the algebra $A$ is semisimple, regular and the set $E_{u}$ is a set of synthesis, then $I_{u}=k\left(E_{u}\right)$, where $k\left(E_{u}\right)=\left\{a \in A: \widehat{a}=0\right.$ on $\left.E_{u}\right\}$. As usual, $\widehat{a}$ denotes the Gelfand transform of $a$.

The next result, which is of independent interest, gives a rather unexpected necessary and sufficient condition for the space $u . A^{*}$ to be weak ${ }^{*}$ closed in $A^{*}$. We first recall that a bounded net $\left(v_{i}\right)_{i \in I}$ in a commutative Banach algebra $B$ is said to be a weak BAI if, for each $b \in B, b v_{i} \rightarrow b$ weakly.

Proposition 2.5. Let $u \in A^{* *}$ be an idempotent. Then the space $u . A^{*}$ is weak closed in $A^{*}$ iff, for any bounded net $\left(u_{i}\right)_{i \in I}$ in $A$ converging to $u$ in the weak ${ }^{*}$ topology of $A^{* *}$, the net $\left(u_{i}+I_{u}\right)_{i \in I}$ is a weak BAI for the quotient algebra $A / I_{u}$.

Proof. Suppose first that the space $u . A^{*}$ is weak ${ }^{*}$ closed in $A^{*}$ so that $\left(A / I_{u}\right)^{*}=$ $u$. $A^{*}$. Let $\left(u_{i}\right)_{i \in I}$ be a bounded net in $A$ converging to $u$ in the weak ${ }^{*}$ topology of $A^{* *}$. Let $a \in A$ and $f \in A^{*}$. Then

$$
\left\langle\left(a+I_{u}\right)\left(u_{i}+I_{u}\right), u . f\right\rangle=\left\langle a u_{i}, u . f\right\rangle
$$

so that

$$
\left\langle a u_{i}, u . f\right\rangle \rightarrow\langle a u, u . f\rangle=\left\langle a+I_{u}, u . f\right\rangle .
$$

Hence

$$
\left(a+I_{u}\right)\left(u_{i}+I_{u}\right) \rightarrow a+I_{u} \text { weakly in } A / I_{u} .
$$

Conversely, suppose that the net $\left(u_{i}+I_{u}\right)_{i \in I}$ is a weak BAI for the quotient algebra $A / I_{u}$. So, for any $a \in A$ and $f \in I_{u}^{\perp}$, we have

$$
\left\langle\left(a+I_{u}\right)\left(u_{i}+I_{u}\right), f\right\rangle \rightarrow\left\langle a+I_{u}, f\right\rangle=\langle a, f\rangle .
$$

Since we also have

$$
\left\langle\left(a+I_{u}\right)\left(u_{i}+I_{u}\right), f\right\rangle=\left\langle a u_{i}+I_{u}, f\right\rangle=\left\langle a u_{i}, f\right\rangle \rightarrow\langle a u, f\rangle=\langle a, u . f\rangle,
$$

we see that, for all $a \in A$ and $f \in I_{u}^{\perp}$, we have equality $\langle a, f\rangle=\langle a, u$.f $\rangle$. Hence $f=u$.f so that $I_{u}^{\perp}=u . A^{*}$. It follows that the space $u . A^{*}$ is weak* closed in $A^{*}$.

Remarks 2.6. These remarks are aimed at clarifying a few questions the reader may have about the preceding results and also about the next theorem.

a) Let $I$ be a closed ideal of $A$ with a BAI. In general, there are infinitely many idempotents $u$ in $A^{* *}$ such that $I=I_{u}$. To see this, let $d \in I^{* *}$ be a right identity of $I^{* *}$. As seen during the proof of Lemma 2.1, $u=e-e d$ is an idempotent and $I_{u}=I$. If $e_{1}$ is another right identity in $A^{* *}$ and $d_{1}$ is another right identity of 
$I^{* *}$, then the element $u_{1}=e_{1}-e_{1} d_{1}$ is an idempotent in $A^{* *}$ and $I_{u_{1}}=I$. In this connection, see remark c) below.

b) Let $X, Y$ be two Banach spaces and $T: X \rightarrow Y$ be a bounded linear operator. In general, $\operatorname{ker}(T)^{* *} \varsubsetneqq \operatorname{ker}\left(T^{* *}\right)$. The equality $\operatorname{ker}(T)^{* *}=\operatorname{ker}\left(T^{* *}\right)$ holds iff $\overline{T^{*}\left(Y^{*}\right)}=\overline{T^{*}\left(Y^{*}\right)} w^{*}$. By Theorem (A) recalled in Section 1, this last equality is satisfied if the range of $T$ is closed. Now let $u \in A^{* *}$ be an idempotent and $T: A \rightarrow A^{* *}$ be the bounded linear operator defined by $T(a)=a u$. The space $u . A^{*}$ is norm closed in $A^{*}$, but this is not the range of $T^{*}$. The range of $T^{*}$ is the space $T^{*}\left(A^{* * *}\right)$, which is in general much larger than the space $T^{*}\left(A^{*}\right)$. The space $T^{*}\left(A^{*}\right)$ is just $u . A^{*}$. The kernel of the operator $T$ is the ideal $I_{u}$ but that of $T^{* *}$ is the left ideal $K_{u}=\left\{m \in A^{* *}: m u=0\right\}$ of $A^{* *}$. In general, $I_{u}^{* *} \subsetneq K_{u}$. These two spaces are equal only if the space $u . A^{*}$ is weak* closed in $A^{*}$.

c) Let $u$ and $v$ be two idempotents in $A^{* *}$ and $T$ and $S$ be two linear operators from $A$ into $A^{* *}$ defined by $T(a)=a u$ and $S(a)=a v$, respectively. If we have $I_{u}=I_{v}$, then this means that $\operatorname{ker}(T)=\operatorname{ker}(S)$. The equality $\operatorname{ker}(T)=\operatorname{ker}(S)$ does not imply the equality $T^{*}\left(A^{* * *}\right)=S^{*}\left(A^{* * *}\right)$, nor the equality $T^{*}\left(A^{*}\right)=S^{*}\left(A^{*}\right)$. It is quite possible that one of the spaces $u . A^{*}$ or $v \cdot A^{*}$ is weak* closed; the other is not.

d) Let $G$ be a nondiscrete nonamenable locally compact group and $A=A(G)+$ $\mathbb{C} 1$, the unitization of the Fourier algebra $A(G)$. Let $u \in A(G)^{* *}$ be a topological invariant mean [33, Theorem 4]. Then $u$ is an idempotent and, for any $a \in A(G)$, $a u=a(\varepsilon) u$, where $\varepsilon$ is the unit element of the group $G$. Hence the ideal $I_{u}$ associated to $u$ is $I_{u}=\{a \in A(G): a(\varepsilon)=0\}$. Since $G$ is not amenable, the ideal $I_{u}$ does not have a BAI [25, Corollary 4.11]. This provides an easy example of a unital commutative Banach algebra $A$ and an idempotent $u \in A^{* *}$ such that the ideal $I_{u}$ does not have a BAI.

e) Let $G$ be an amenable connected group, $A=A(G)$ and $J=k(F)$ for some nonempty closed set in the coset ring $\Re\left(G_{d}\right)$ distinct from $G$. The ideal $J$ has a BAI so that $J^{* *}$ has a right unit $u$. Then the set $E_{u}=\{\gamma \in \Delta(A): u(\gamma)=1\}=G \backslash F$ is a nonclosed open subset of $G=\Delta(A)$. So, since the set $E_{u}=u . A^{*} \cap \Delta(A)$ is not closed in $\Delta(A)$, the space $u . A^{*}$, which is norm closed, is not weak* closed in $A^{*}=V N(G)$.

f) In Proposition 2.5, instead of assuming that "for any bounded net $\left(u_{i}\right)_{i \in I}$ in $A$ converging to $u$ in the weak ${ }^{*}$ topology of $A^{* *} \ldots$.., it is enough to suppose that "for some bounded net $\left(u_{i}\right)_{i \in I}$ in $A$ converging to $u$ in the weak* topology of $A^{* *} \ldots$...

The next theorem, which is the main result of this section, gives a necessary and sufficient condition in terms of idempotent elements of the algebra $A^{* *}$ for a closed ideal $I$ of $A$ to have a BAI. The proof of this theorem does not really use the hypothesis that $A$ is commutative. It is valid for any Banach algebra $A$ provided that $A$ has a BAI and $I$ is a closed ideal with a bounded right approximate identity. For the proof of this theorem we need the following result that we recall now.

Let $B_{1}$ and $B_{2}$ be two Banach algebras such that $B_{1}$ is a subalgebra of $B_{2}$. (For us, $B_{1}$ will be $A$ and $B_{2}=A^{* *}$.) A bounded linear operator $T: B_{1} \rightarrow B_{2}$ is said to be a multiplier if, for each $x$ and $y$ in $B_{1}$, the equalities $x T(y)=T(x y)=T(x) y$ hold. On both $B_{1}^{* *}$ and $B_{2}^{* *}$ we put the first Arens multiplication. Then $B_{1}^{* *}$ is a subalgebra of $B_{2}^{* *}$ and, as the iterated-limits definition of the Arens multiplication shows, $T^{* *}: B_{1}^{* *} \rightarrow B_{2}^{* *}$ is also a multiplier. 
Theorem 2.7. Let I be a closed ideal of $A$. Then the ideal I has a BAI iff there is an idempotent $u \in A^{* *}$ such that $I=I_{u}$ and the subalgebra $A u$ is norm closed in $A^{* *}$.

Proof. Suppose first that the ideal $I$ has a BAI. By Lemmas 2.1 and 2.3, we can choose an idempotent $u \in A^{* *}$ such that $I=I_{u}$ and the space $u . A^{*}$ is weak* closed in $A^{*}$. Let

$$
\phi: A / I_{u} \rightarrow A^{* *}
$$

be the mapping defined by $\phi\left(a+I_{u}\right)=a u$. It is easy to verify that $\phi$ is a well-defined linear operator and it applies in one-to-one manner the quotient algebra $A / I_{u}$ onto the subalgebra $A u$ of $A^{* *}$. The mapping $\phi$ is actually an algebra homomorphism. To prove that the subalgebra $A u$ is closed in $A^{* *}$, we are going to prove that $\phi$ is a normed-space isomorphism from $A / I_{u}$ onto $A u$. We first recall that, by Lemma 2.3, $I_{u}^{\perp}=u . A^{*}$. Hence we can identify the dual space of the Banach algebra $A / I_{u}$ with $u$. $A^{*}$. It follows that, for a given $a \in A$ and all $f \in A^{*}$,

$$
\left\|a+I_{u}\right\|=\operatorname{Sup}_{\|u . f\| \leq 1}\left|\left\langle a+I_{u}, u . f\right\rangle\right|=\operatorname{Sup}_{\|u . f\| \leq 1}|\langle a u, f\rangle| .
$$

Since the space $u . A^{*}$ is closed in $A^{*}$, by the Open Mapping Theorem applied to the bounded linear operator $f \mapsto u$. $f$, there is a constant $\beta>0$ such that

$$
u . A_{1}^{*} \supseteq \beta .\left(u \cdot A^{*}\right)_{1} .
$$

Hence, for all $f \in A^{*}$,

$$
\begin{aligned}
\left\|a+I_{u}\right\| & =\operatorname{Sup}_{\|u . f\| \leq 1}\left|\left\langle a+I_{u}, u . f\right\rangle\right|=\operatorname{Sup}_{\|u . f\| \leq 1}|\langle a u, f\rangle| \\
& =\operatorname{Sup}_{\|u . f\| \leq 1}|\langle a, u . f\rangle| \leq \frac{1}{\beta} \operatorname{Sup}_{\|f\| \leq 1}|\langle a u, f\rangle|=\frac{1}{\beta} .\|a u\|
\end{aligned}
$$

so that

$$
\left\|a+I_{u}\right\| \leq \frac{1}{\beta} \cdot\|a u\|
$$

On the other hand, since $\|u . f\| \leq\|u\| .\|f\|$, for $f$ in $A^{*}$,

$$
\begin{aligned}
\|a u\| & =\operatorname{Sup}_{\|f\| \leq 1}|\langle a u, f\rangle|=\operatorname{Sup}_{\|f\| \leq 1}\left|\left\langle a+I_{u}, u . f\right\rangle\right| \\
& \leq\left\|a+I_{u}\right\| . \operatorname{Sup}_{\|f\| \leq 1}\|u . f\| \leq\left\|a+I_{u}\right\| \cdot\|u\|,
\end{aligned}
$$

we obtain the inequality

$$
\|a u\| \leq\|u\| \cdot\left\|a+I_{u}\right\| .
$$

The inequalities (1) and (2) show that both the operator $\phi$ and its inverse are bounded. Hence $\phi$ is an isomorphism. Since the quotient algebra $A / I_{u}$ is Banach, so is the algebra $A u$. It follows that the subalgebra $A u$ is closed in $A^{* *}$.

Conversely, suppose that, for some idempotent $u \in A^{* *}, I=I_{u}$ and that the subalgebra $A u$ is closed in $A^{* *}$. We are going to prove that the space $u$. $A^{*}$ is weak* closed in $A^{*}$. To this end, let $T: A \rightarrow A^{* *}$ be the mapping defined by $T(a)=a u$. This is a bounded Banach algebra homomorphism with closed range. Then, by Theorem (A) in Section 1, $T^{*}\left(A^{* * *}\right)$ is weak* closed in $A^{*}$. The kernel of $T$ is the ideal $I_{u}$ so that $T^{*}\left(A^{* * *}\right)=I_{u}^{\perp}$. Our aim is to show that $T^{*}\left(A^{* * *}\right)=u$. $A^{*}$. Since, for each $f \in A^{*}, T^{*}(f)=u$. $f$, the inclusion $u . A^{*} \subseteq T^{*}\left(A^{* * *}\right)$ is clear.

To prove the reverse inclusion, it is enough to prove that, for $F \in A^{* * *}$, one has $u . T^{*}(F)=T^{*}(F)$. To this end, observe that the homomorphism $T: A \rightarrow A^{* *}$, 
$T(a)=a u$, is a multiplier as well. Then, since $T^{* *}: A^{* *} \rightarrow A^{* * * *}$ is also a multiplier, for $a \in A$, we have

$$
T^{* *}(a u)=T^{* *}(a) u=T(a) u=a u u=a u=T(a) .
$$

Hence, for $F \in A^{* * *}$, we have the equalities

$$
\begin{aligned}
\left\langle a, u . T^{*}(F)\right\rangle & =\left\langle a u, T^{*}(F)\right\rangle=\left\langle T^{* *}(a u), F\right\rangle \\
& =\langle a u, F\rangle=\langle T(a), F\rangle=\left\langle a, T^{*}(F)\right\rangle .
\end{aligned}
$$

Since $T^{*}(F) \in A^{*}$, from the equality $\left\langle a, u \cdot T^{*}(F)\right\rangle=\left\langle a, T^{*}(F)\right\rangle$, which is valid for any $a \in A$, we conclude that $u \cdot T^{*}(F)=T^{*}(F)$. Thus $T^{*}\left(A^{* * *}\right)=u \cdot A^{*}$, and the space $u . A^{*}$ is weak ${ }^{*}$ closed in $A^{*}$. It follows, by Lemma 2.3 , that the ideal $I_{u}$ has a BAI.

The isomorphism $\phi: A / I_{u} \rightarrow A, \phi\left(a+I_{u}\right)=a u$, which has appeared in the proof of the preceding theorem, is also $A$-invariant (i.e. an $A$-module isomorphism) in the sense that, for all $a, b$ in $A$, the equality

$$
\phi\left(a b+I_{u}\right)=a \phi\left(b+I_{u}\right)
$$

holds. It happens that, for any closed ideal $I$ of $A$, the only $A$-invariant isomorphisms from $A / I$ into $A^{* *}$ are of this form. Actually we have such an isomorphism iff the ideal $I$ has a BAI. This is the content of the next corollary, which is the second main result of this section.

Corollary 2.8. A closed ideal I of $A$ has a BAI iff there is an A-invariant isomorphism from the quotient algebra $A / I$ into $A^{* *}$.

Proof. If the ideal $I$ has a BAI, then $I=I_{u}$ for some idempotent $u \in A^{* *}$ for which the subalgebra $A u$ is closed in $A^{* *}$. Then, as observed above, there is an $A$-invariant isomorphism from the algebra $A / I_{u}$ into $A^{* *}$, namely the mapping $a+I_{u} \mapsto a u$. Conversely, suppose that there is an $A$-invariant isomorphism $\phi: A / I \rightarrow A^{* *}$. Let $\left(e_{j}\right)_{j \in J}$ be a BAI in $A$ that converges in the weak* topology of $A^{* *}$ to $e$. The net $\left(e_{j}+I\right)_{j \in J}$ is bounded in $A / I$. If necessary, replacing it by a subnet of it, we can assume that $u=w^{*}-\lim _{j} \phi\left(e_{j}+I\right)$ exists in $A^{* *}$. Since, for $a \in A$,

$$
(a+I)\left(e_{j}+I\right)=a e_{j}+I \rightarrow a+I
$$

in the norm topology of $A / I$ and since $\phi$ is $A$-invariant, applying $\phi$ to both sides of the preceding equality, we see that $\phi(a+I)=a u$. Hence, since $\phi$ is an isomorphism and $\phi(A / I)=A u$, the subalgebra $A u$ is closed in $A^{* *}$. On the other hand, since $\phi$ is an isomorphism and $\phi(a+I)=a u$, we see that $a u=0$ iff $a \in I$, so that $I=I_{u}$. It remains to show that $u$ is an idempotent. To see this we first remark that, in the equality $\phi(a+I)=a u$, replacing $a$ by $e_{j}$ and passing to the limit in the weak* topology of $A^{* *}$, we get that $e u=u$. Now for $i \neq j$,

$$
e_{i} \phi\left(e_{j}+I\right)=\phi\left(e_{i} e_{j}+I\right)=\phi\left(e_{i}+I\right) \phi\left(e_{j}+I\right)=e_{i} e_{j} u^{2} .
$$

In these equalities, passing to the limit first in $j$ and then in $i$ (both in the weak* topology of $A^{* *}$ ), we obtain that $u=u^{2}$ so that $u$ is an idempotent. Hence, since $A u$ is closed in $A^{* *}$, by the preceding theorem, we conclude that the ideal $I$ has a BAI. 
This corollary shows that, when the ideal $I$ has a BAI, one can realize the quotient algebra $A / I$ as a subalgebra of $A^{* *}$ in a very simple way, as $A u$ for some idempotent $u \in A^{* *}$. Thus the quotient space $A / I$ possesses all the hereditary properties of the Banach space $A^{* *}$. Moreover, working with the algebra $A u$ is apparently easier than working with the quotient algebra $A / I$. The dual space of the algebra $A u$ is also easy to work with; it is (up to a natural isomorphism) just u. $A^{*}$.

As an immediate consequence of this corollary, we present the following result.

Corollary 2.9. Let $B$ be a Banach algebra and $\phi: A \rightarrow B$ a bounded surjective homomorphism. If $\operatorname{ker}(\phi)$ has a BAI, then the algebra $B$ is isomorphic to a subalgebra of $A^{* *}$.

There is a case where the closedness of the subalgebra $A u$ in $A^{* *}$ is automatic, whatever the idempotent $u \in A^{* *}$ is. This is the case when the algebra $A$ is an ideal in its second dual. It is well known and easy to prove that $A$ is an ideal in $A^{* *}$ iff, for each $a \in A$, the multiplication operator $b \mapsto a b$ is weakly compact. If this is the case, for any $u \in A^{* *}, A u \subseteq A$. For instance, for any discrete group $G$, the Fourier algebra $A(G)$ is an ideal in its second dual since it is generated by its idempotent elements. If the algebra $A$ is an ideal in its second dual, then, since for any idempotent $u \in A^{* *}$ one has $A u \subseteq A$, the linear operator $T: A \rightarrow A$, defined by $T(a)=a u$, is a projection and also a multiplier on $A$. Hence $A u$ is closed in $A$, and so in $A^{* *}$, and $A=T(A) \oplus(I-T)(A)$. It follows that the ideal $I_{u}=(I-T)(A)$ has a BAI. The projection $P=T^{*}$ is obviously an invariant projection from $A^{*}$ onto $I_{u}^{\perp}$. We record this observation.

Corollary 2.10. If the algebra $A$ is an ideal in its second dual $A^{* *}$, then, for any idempotent $u \in A^{* *}$, the ideal $I_{u}$ has a BAI.

Remark 2.11. We recall here a classical result about function algebras because of its similarity to Corollary 2.8 above. To recall this result, let $\Omega$ be a compact Hausdorff space and $B$ a closed unital subalgebra of $C(\Omega)$ that separates the points of $\Omega$. A closed subset $F$ of $\Omega$ is said to be a peak set for $B$ if there is a function $a \in B$ such that $a(x)=1$ for $x \in F$ and $|a(x)|<1$ for $x \in \Omega \backslash F$. Now let $F$ be the intersection of a family of peak sets. Then the closed ideal $k(F)=\{a \in B: a=0$ on $F\}$ has a BAI $[29$, p. 160, Theorem 1] and the "restriction algebra" of $B$ to $F$, $B \mid F=\{a \mid F: a \in B\}$, is closed in $C(F)$. Further, the mapping $\phi: B / k(F) \rightarrow C(F)$, defined by $\phi(a+k(F))=a \mid F$, is an isomorphism from $B / k(F)$ onto $B \mid F[29$, p. 163, Theorem 3]. In Theorem 2.7 and Corollary 2.8, supposing that $A$ is semisimple and interpreting the algebra $A u$ as the restriction algebra of $A$ to the set $E_{u}$, Corollary 2.8 might be thought of as a generalization of this classical result.

The final result of this section presents a simple way to check that the subalgebra $A u$ is closed in $A^{* *}$. In this proposition $u \in A^{* *}$ is an arbitrary idempotent and $\left(u_{i}\right)_{i \in I}$ is an arbitrary bounded net in $A$ that converges in the weak ${ }^{*}$ topology of $A^{* *}$ to $u$. Note that the net $\left(u_{i} u\right)_{i \in I}$ is a bounded net in the algebra $A u$.

Proposition 2.12. The subalgebra $A u$ is closed in $A^{* *}$ iff the net $\left(u_{i} u\right)_{i \in I}$ is a weak BAI for the algebra Au.

Proof. Suppose first that the algebra $A u$ is closed in $A^{* *}$. Then the mapping $\phi: A / I_{u} \rightarrow A^{* *}$, defined by $\phi\left(a+I_{u}\right)=a u$, is an isomorphism from $A / I_{u}$ onto $A u$. Hence, as seen during the proof of Theorem 2.7, the space $u . A^{*}$ is weak* closed in 
$A^{*}$. So, by Proposition 2.5, the net $\left(u_{i}+I_{u}\right)_{i \in I}$ is a weak BAI for the quotient algebra. Hence the net $\left(\phi\left(u_{i}+I_{u}\right)\right)_{i \in I}$, that is, the net $\left(u_{i} u\right)_{i \in I}$, is a weak BAI for the algebra $A u$.

Conversely, suppose that the net $\left(u_{i} u\right)_{i \in I}$ is a weak BAI for the algebra $A u$. Let $T: A \rightarrow A^{* *}$ be the linear operator defined by $T(a)=a u$. To prove that $A u$ is closed in $A^{* *}$, it is enough, by Theorem (A) in Section 1, to prove that $T^{*}\left(A^{* * *}\right)$ is norm closed in $A^{*}$. Since $u$ is an idempotent, the space $u . A^{*}$ is norm closed in $A^{*}$ and $u . A^{*} \subseteq T^{*}\left(A^{* * *}\right)$. So it is enough to prove that $T^{*}\left(A^{* * *}\right)=u \cdot A^{*}$. To prove this, we will again prove that, for $F \in A^{* *}, u \cdot T^{*}(F)=T^{*}(F)$. By hypothesis, for $a \in A$, the net $\left(u_{i} u . a u\right)_{i \in I}$ converges weakly in $A^{* *}$ to $a u$. Hence, for $F \in A^{* * *}$ and $T(a)=a u$, we have

$$
\left\langle u_{i} u . a u, F\right\rangle=\left\langle u_{i} a u, F\right\rangle=\left\langle T\left(u_{i} a\right), F\right\rangle=\left\langle a u_{i}, T^{*}(F)\right\rangle .
$$

Passing to the limit, we obtain that

$$
\langle a u, F\rangle=\left\langle a u, T^{*}(F)\right\rangle .
$$

Since $\langle a u, F\rangle=\langle T(a), F\rangle=\left\langle a, T^{*}(F)\right\rangle$, we see that, for all $a \in A,\left\langle a u, T^{*}(F)\right\rangle=$ $\left\langle a, T^{*}(F)\right\rangle$. Since $T^{*}(F)$ is in $A^{*}$, we conclude that $u \cdot T^{*}(F)=T^{*}(F)$. Hence $T^{*}\left(A^{* * *}\right)=u \cdot A^{*}$ so that $T^{*}\left(A^{* * *}\right)$ is norm closed in $A^{*}$. It follows that $T(A)=A u$ is closed in $A^{* *}$.

\section{NATURAL PROJECTIONS VERSUS INVARIANT PROJECTIONS}

In this section $A$ will again be a commutative Banach algebra with a BAI. To avoid the trivial cases, we assume that $A$ is semisimple. As above, by $e$ we always denote a right identity in $A^{* *}$.

We recall that a projection $P: A^{*} \rightarrow A^{*}$ is said to be invariant if $P(a . f)=a . P(f)$ for all $a \in A$ and $f \in A^{*}$. In this section our main problem is the second aspect of Problem (A). Namely,

Problem (B): If a weak ${ }^{*}$ closed invariant subspace $X$ of $A^{*}$ is complemented in $A^{*}$, then when is $X$ invariantly complemented in $A^{*}$ ?

In this section we present a few results related to this problem. The main result of this section is Theorem 3.3.

Now let $X$ be a weak* closed invariant subspace of $A^{*}$. The spectrum of $X$, $S p(X)$, by definition, is the set $S p(X)=X \cap \Delta(A)$. If $P: A^{*} \rightarrow X$ is any projection from $A^{*}$ onto $X$, then, since $X=P\left(A^{*}\right)$, for any $\gamma \in X \cap \Delta(A), P(\gamma)=\gamma$. However, for $\gamma$ in the set $\Delta(A) \backslash S p(X)$, whether $P(\gamma)=0$ or not depends on the projection. This fact led us to introduce the following definition.

Definition 3.1. We say that a projection $P: A^{*} \rightarrow A^{*}$ is "natural" if, for each $\gamma \in \Delta(A)$, either $P(\gamma)=\gamma$ or $P(\gamma)=0$.

If $X$ is a closed invariant subspace of $A^{*}$ and if there is a natural projection $P$ from $A^{*}$ onto $X$, we shall say that $X$ is "naturally complemented" in $A^{*}$. We shall see below that every invariant projection is natural. We do not know whether every natural projection is invariant. These two classes of projections do not seem to be the same. However, as we shall see below, in some important cases, to show that a closed ideal $I$ of $A$ has a BAI it is enough to show that $I^{\perp}$ is naturally complemented in $A^{*}$. 
Lemma 3.2. Let $P: A^{*} \rightarrow A^{*}$ be a projection. Then

a) $P$ is natural iff, for each $\gamma \in \Delta(A)$ and $a \in A, P(a . \gamma)=a \cdot P(\gamma)$.

b) Every invariant projection $P: A^{*} \rightarrow A^{*}$ is natural.

c) If $P: A^{*} \rightarrow A^{*}$ is a natural projection, then for $f$ in $\overline{\operatorname{Span}(\Delta(A))}$ and $a \in A$, $P(a . f)=a \cdot P(f)$.

Proof. a) Suppose first that $P: A^{*} \rightarrow A^{*}$ is a natural projection. Let $\gamma \in \Delta(A)$ and $a \in A$. As $a . \gamma=\langle a, \gamma\rangle \gamma$, we have $P(a . \gamma)=\langle a, \gamma\rangle P(\gamma)$. If $P(\gamma)=\gamma$, then $\langle a, \gamma\rangle P(\gamma)=a \cdot \gamma=a . P(\gamma)$ so that $P(a . \gamma)=a . P(\gamma)$. If $P(\gamma)=0$, then again $P(a . \gamma)=\langle a, \gamma\rangle P(\gamma)=0=a . P(\gamma)$. Thus, for any $\gamma \in \Delta(A)$ and $a \in A$, $P(a . \gamma)=a \cdot P(\gamma)$

Conversely, suppose that for each $\gamma \in \Delta(A)$ and $a \in A$, we have $P(a \cdot \gamma)=a P(\gamma)$. Let $\gamma \in \Delta(A)$ be such that $P(\gamma) \neq 0$. Then, since for $a \in A, a \cdot \gamma=\langle a, \gamma\rangle \gamma$ and $P(a . \gamma)=a . P(\gamma)$, we get the equalities

$$
a . P(\gamma)=P(a . \gamma)=\langle a, \gamma\rangle P(\gamma) .
$$

Applying $e$ to both sides of these equalities, we get the equality

$$
\langle a, P(\gamma)\rangle=\langle a, \gamma\rangle \cdot\langle e, P(\gamma)\rangle
$$

so that

$$
P(\gamma)=\langle e, P(\gamma)\rangle \gamma
$$

Since we have supposed that $P(\gamma) \neq 0$, the above equality shows that $\langle e, P(\gamma)\rangle \neq 0$. Applying $P$ to both sides of this equality, we get that

$$
P(\gamma)=\langle e, P(\gamma)\rangle^{2} \gamma=\langle e, P(\gamma)\rangle \gamma
$$

so that $\langle e, P(\gamma)\rangle=1$. Hence $P(\gamma)=\gamma$. This shows that $P$ is natural.

b) Assertion b) is immediate from a).

c) Let $P$ be a natural projection on $A^{*}$. Let $f=\sum_{i=1}^{n} c_{i} \gamma_{i}$ be an element in the space $\operatorname{Span}(\Delta(A))$. Put $F=\left\{i: 1 \leq i \leq n, P\left(\gamma_{i}\right) \neq 0\right\}$. Then $P(f)=\sum_{i \in F} c_{i} \gamma_{i}$. On the other hand, for any $a \in A, a . f=\sum_{i=1}^{n} c_{i}\left\langle a, \gamma_{i}\right\rangle \gamma_{i}$ so that

$$
P(a . f)=\sum_{i \in F} c_{i}\left\langle a, \gamma_{i}\right\rangle \gamma_{i}=a . P(f) .
$$

The preceding line, by a simple passage to the limit, implies that the equality $P(a . f)=a . P(f)$ holds for any $f$ in the space $\overline{\operatorname{Span}(\Delta(A))}$ too. Hence $P$, as a mapping from $\overline{\operatorname{Span}(\Delta(A))}$ into itself, is invariant.

If the algebra $A$ is an ideal in its second dual, then, since the algebra $A$ is semisimple, as a Hahn-Banach argument will show, $\overline{\operatorname{Span}(\Delta(A))}=A A^{*}$. So, in this case, any natural projection $P$ on $A^{*}$ maps $A A^{*}$ into itself and, as a mapping from $A A^{*}$ into itself, is invariant. Moreover, in this case the projection $Q: A^{*} \rightarrow A^{*}$, defined by $\langle Q(f), a\rangle=\langle P(a . f), e\rangle$, is an invariant projection on $A^{*}$ so that in this special case the invariant projections and the natural projections are essentially the same.

The natural projections are very closely related to the following very weak notion of BAI. Let $I$ be a closed ideal of $A$. We say that the ideal $I$ has a $\Delta$-weak BAI if $I$ contains a bounded net $\left(u_{i}\right)_{i \in I}$ such that, for each $\gamma \in \Delta(I),\left\langle u_{i}, \gamma\right\rangle \rightarrow 1$. This kind of BAI was introduced by Jonas and Lahr in [20]. For this reason at some places it is also called "weak BAI in the sense of Jonas and Lahr". As proved in [20] and [19], existence of a $\Delta$-weak BAI in a commutative semisimple Banach 
algebra $B$ does not imply that the algebra $B$ has an approximate identity, even an unbounded one. In the paper 19] the authors exhibit two classes of Segal algebras with $\Delta$-weak BAIs. These Segal algebras do not have BAI. We shall need to use this kind of weak BAI in the proof of the next result.

As mentioned in the introduction, a closed ideal $I$ of the group algebra $L^{1}(G)$ of a locally compact abelian group $G$ has a BAI iff its annihilator $X=I^{\perp}$ in $L^{\infty}(G)$ is complemented in $L^{\infty}(G)$ ([32, Theorem 1.1] and [14, Theorem A]). As far as we know, it is not known whether the same result is true for the closed ideals of the Fourier algebra $A(G)$ of a locally compact amenable group $G$. In the next result we prove that a closed ideal $I$ of $A(G)$ has a BAI iff the invariant space $I^{\perp}$ is naturally complemented in $V N(G)$. For results involving the operator space structure of $A(G)$ and "completely bounded projections", see [6] and 39].

Theorem 3.3. Let $G$ be an amenable locally compact group and I a closed ideal of the Fourier algebra $A=A(G)$ of $G$. Then the following assertions are equivalent:

a) The invariant subspace $X=I^{\perp}$ is invariantly complemented in $A^{*}$.

b) The space $X=I^{\perp}$ is naturally complemented in $A^{*}$.

c) The spectrum $S p(X)$ of $X$ is in the closed coset ring $\Re_{c}\left(G_{d}\right)$.

Proof. Implication a) $\Rightarrow$ b) follows from Lemma 3.2 above. To prove implication $\mathrm{b}) \Rightarrow \mathrm{c}$ ), suppose that $I^{\perp}$ is naturally complemented in $A^{*}=V N(G)$. Let $P: A^{*} \rightarrow$ $I^{\perp}$ be a natural projection. Set $E=I^{\perp} \cap \Delta(A)$. We identify $\Delta(A)$, via point evaluations, with $G$. Since the space $I^{\perp}$ is weak* closed in $A^{*}$, the set $E$ is closed in $G$ and is the hull of the ideal $I$. Since $E \subseteq P\left(A^{*}\right)$, for $\gamma \in E, P(\gamma)=\gamma$. For $\gamma \in G \backslash E$, since $P$ is a natural projection, necessarily, $P(\gamma)=0$. Put $u=e-P^{*}(e)$. As

$$
I^{\perp \perp}=P\left(A^{*}\right)^{\perp}=\operatorname{ker}\left(P^{*}\right)=\left(I-P^{*}\right)\left(A^{* *}\right),
$$

$u \in I^{\perp \perp}$. Identifying $I^{\perp \perp}$ with $I^{* *}$, we get that $u \in I^{* *}$. Let $\left(u_{i}\right)_{i \in I}$ be a bounded net in $I$ that converges in the weak* topology of $A^{* *}$ to $u$. Then this net $\left(u_{i}\right)_{i \in I}$ is a $\Delta$-weak BAI for the ideal $I$. Indeed, the Gelfand spectrum of the ideal $I$ is the set $G \backslash E$ and, for $\gamma \in G \backslash E$, since $P(\gamma)=0$, we have

$$
\lim _{i}\left\langle u_{i}, \gamma\right\rangle=\langle u, \gamma\rangle=\left\langle e-P^{*}(e), \gamma\right\rangle=\langle e, \gamma\rangle=1 .
$$

Thus, the ideal $I$ has a $\Delta$-weak BAI. Now consider $\left(u_{i}\right)_{i \in I}$ as a net in the algebra $B\left(G_{d}\right)$, the Fourier-Stieltjes algebra of $G_{d}$. Since the embedding of $A(G)$ into $B\left(G_{d}\right)$ is isometric, the net $\left(u_{i}\right)_{i \in I}$ is bounded in $B\left(G_{d}\right)$. Let $w$ be a $\sigma\left(B\left(G_{d}\right), C^{*}\left(G_{d}\right)\right)$ cluster point of this net. Then $w$ is an idempotent and its support is the set $E$. Hence, since the set $E$ is closed, by Host's idempotent theorem [13, $E \in \Re_{c}\left(G_{d}\right)$.

Finally, to prove that c) implies a), we recall that, by [9], any set $E$ in the ring $\Re_{c}\left(G_{d}\right)$ is a set of synthesis and the ideal $k(E)$ has a BAI. So, since $S p(X)$ is in the ring $\Re_{c}\left(G_{d}\right)$ and $X=I^{\perp}$, the ideal $I$ has a BAI and, by Lemma 2.2, there is an invariant projection from $A^{*}$ onto $I^{\perp}$. This completes the proof.

The proof of the preceding theorem shows that the following general result holds.

Proposition 3.4. Let $I$ be a closed ideal of the algebra $A$. If $I^{\perp}$ is naturally complemented in $A^{*}$, then I has a $\Delta$-weak BAI. 


\section{Applications}

In this section we are going to present some applications of the results obtained in Section 2, especially of Corollary 2.8. As in Section 2, in this section $A$ is again an arbitrary commutative Banach algebra with a BAI.

a) Weak sequential completeness of the quotient space $A / I$. As is well known, any separable Banach space is isomorphic to a quotient space of the sequence space $\ell^{1}$, a weakly sequentially complete Banach space (=WSC). This result shows that almost none of the Banach space properties of $\ell^{1}$ pass to its quotient space. In contrast with this result, an important result in the interface of complex analysis and harmonic analysis, known as the Havin-Moorey Theorem, states that the quotient space $L^{1}(T) / k(-\mathbb{N})$ is WSC [12, p. 47]. Here $T=\{z \in$ $\mathbb{C}:|z|=1\}$ is the unit circle group and $k(-\mathbb{N})=\left\{a \in L^{1}(T): \widehat{a}(n)=0\right.$ for $n<0\}$. In the papers [16] and [17] Godefroy made a deep study of the closed ideals of the algebra $L^{1}(G)$ ( $G$ is compact and abelian) and the closed subspaces of the Lebesgue space $L^{1}(\mu)$ of a general $\sigma$-finite measure space for which the corresponding quotient spaces are WSC. His main ingredient is the fact that any $L^{1}$ space is $\ell^{1}$-complemented in its second dual. In this section we are going to present some cases where the quotient algebra $A / I$ is WSC.

A Banach algebra $A$ with a BAI has in general many proper subalgebras $B$ with a BAI. For instance, if $\phi$ is a bounded homomorphism from $A$ into itself, then the subalgebra $B=\overline{\phi(A)}$ has a BAI. The Banach spaces properties of $A$ and $B$ are very different in general. For instance, even if $A$ is $\ell^{1}$-complemented in its second dual, $B$ need not be $\ell^{1}$-complemented in its second dual. However, since $B^{* *}$ is a closed subalgebra of $A^{* *}$, the space $B^{* *}$ is WSC if $A^{* *}$ is so. Besides the WSC'ness, we could also consider other geometric properties on $A^{* *}$, but we shall not consider them here.

The result below is quite general since the only hypothesis put on the algebra $A$ is the existence of a BAI. This result is an immediate consequence of Corollary 2.8.

Theorem 4.1. If the second dual $A^{* *}$ of the algebra $A$ is WSC, then for any closed ideal I of $A$ with BAI, the quotient space $A / I$ is also WSC.

This theorem is valid even if the algebra $A$ is not commutative, provided that $I$ is an ideal with a bounded right approximate identity. As proved in [23], every homomorphism $\phi: L^{1}(G) \rightarrow L^{1}(G)$ (G is locally compact abelian) has closed range. So, if $\operatorname{ker}(\phi)$ has a BAI, $\phi\left(L^{1}(G)\right)$ is isomorphic to a subalgebra of the form $L^{1}(G) u$ of $L^{1}(G)^{* *}$ for some idempotent $u \in L^{1}(G)^{* *}$. Also, for instance, if $\phi: L^{1}(G) \rightarrow L^{1}(G)$ is a homomorphism and $I$ is a closed ideal of the subalgebra $\phi\left(L^{1}(G)\right)$ with a BAI, then the quotient space $\phi\left(L^{1}(G)\right) / I$ is also WSC. As another immediate application of the above theorem, we give the following result.

Corollary 4.2. Let $G$ be a locally compact amenable group and $\phi$ a bounded homomorphism from $A(G)$ into some Banach algebra $B$ such that $\operatorname{ker}(\phi)$ has a BAI. Then, unless $B$ is WSC, $\phi$ cannot be surjective.

In the case where $G$ is amenable, as mentioned above, the closed ideals with BAI of the algebra $A(G)$ have been characterized in the paper 9]. These are exactly the ideals $k(E)$, where $E$ is a closed subset of $G$ that belongs to the coset ring $\Re\left(G_{d}\right)$. As mentioned in the introduction, for $G$ abelian, the structure of the closed elements of the ring $\Re\left(G_{d}\right)$ are described by Gilbert [14] and Schreiber [38]. Forrest 
[7] verified that the same description holds in the nonabelian case too, so that the structure of these sets is explicitly known. As another application of Corollary 2.8, we give the following result.

Corollary 4.3. a) Let $G$ be a locally compact amenable group and $A=A(G)$ its Fourier algebra. For any closed set $E$ belonging to the ring $\Re\left(G_{d}\right)$, the quotient algebra $A(G) / k(E)$ is $W S C$.

b) For any locally compact group $G$ and for any closed ideal $I$ of $B(G)$ with a $B A I$, the quotient space $B(G) / I$ is weakly sequentially complete.

As proved in [22], for any power bounded element $u$ of $B(G)$, the ideal $I=$ $\overline{(1-u) B(G)}$ has a BAI. So, for any power bounded element $u$ of $B(G)$, the quotient space $B(G) / \overline{(1-u) B(G)}$ is WSC.

Let $G$ be a locally compact abelian group and $E$ be a closed subset of $\widehat{G}$. We recall that if the restriction of the Fourier transform, $\phi \mid E: L^{1}(G) \rightarrow C_{0}(E)$, defined by $\phi|E(a)=\widehat{a}| E$, is surjective, the set $E$ is said to be a Helson set (see [35, Chapter $5]$ ). The kernel of the homomorphism $\phi \mid E$ is the ideal $k(E)$. Thus, if $E$ is a Helson set, the Banach algebras $L^{1}(G) / k(E)$ and $C_{0}(E)$ are isomorphic. The next result, which is an application of Corollary 4.2, implies that the ideal $k(E)$ cannot have a BAI unless the set $E$ is finite.

Corollary 4.4. Suppose that $A^{* *}$ is WSC, $B$ is a $C^{*}$-algebra and $\phi: A \rightarrow B$ a bounded surjective homomorphism. Then the ideal $\operatorname{ker}(\phi)$ cannot have a BAI unless its codimension is finite.

Proof. If $\operatorname{ker}(\phi)$ has a BAI, then, by Theorem $4.1, B$ is WSC. However, a $C^{*}$-algebra cannot be WSC unless it is finite dimensional. The conclusion follows.

Finally, in passing we mention the following variant of Theorem 4.1. To this purpose, let $m \in A^{* *}$ be an arbitrary element. Then the subspace $m . A^{*}$ is an invariant subspace of $A^{*}$ but it need not be norm closed. On the other hand, the set $I_{m}=\{a \in A: a m=0\}$ is a closed ideal of $A$ but the space $A m$ need not be a subalgebra of $A^{* *}$. If $m . A^{*}$ is weak* closed in $A^{*}$, then $I_{m}^{\perp}=m . A^{*}$. In this case, as the first part of the proof of Theorem 2.7 shows, the subspace $A m$ of $A^{* *}$ is closed in $A^{* *}$ and the quotient space $A / I_{m}$ is isomorphic to $A m$. So, if the space $m . A^{*}$ is weak* closed in $A^{*}$, the quotient space $A / I_{m}$ has all the hereditary properties of the Banach space $A^{* *}$.

b) Multipliers of the quotient algebra $A / I$. Let $I$ be a closed ideal with a BAI of $A$. As the second application of Corollary 2.8, in this subsection we consider the problem of determination of the multipliers of the quotient algebra $A / I$. As far as we were able to determine, there is only one paper dealing with this problem in the literature. This is a paper by Glicksberg and Wik [15], where the authors consider the problem of determination of the multipliers of certain quotient algebras of the group algebra $L^{1}(G)$ of a locally compact abelian group $G$. We need some preliminary results about the multipliers of a commutative semisimple Banach algebra.

Let $B$ be a commutative semisimple Banach algebra. There are two equivalent definitions of multipliers on $B$ :

a) A (necessarily bounded) linear operator $T: B \rightarrow B$ is said to be a multiplier if, for each $a, b$ in $B$, the equalities $a T(b)=T(a b)=T(a) b$ hold. 
b) A (necessarily bounded and continuous) function $\varphi: \Delta(B) \rightarrow \mathbb{C}$ is said to be a multiplier of the algebra $B$ if $\varphi \widehat{B} \subseteq \widehat{B}$.

For more information on multipliers of Banach algebras we refer the reader to the book [27]. We shall work with the second definition. In this subsection we suppose that the algebra $A$ is semisimple. Let $I$ be a closed ideal of $A$ with a BAI. We choose an idempotent $u \in A^{* *}$ such that $I=I_{u}$ and the space $u$. $A^{*}$ is weak* closed in $A^{*}$. Then the set $E=u \cdot A^{*} \cap \Delta(A)$ is closed in $\Delta(A)$ and it is the hull of the ideal $I$. The quotient algebra $A / I$ is semisimple iff $I=k(E)$. In this case the Gelfand spectrum of the quotient algebra $A / I$ is the set $E$. We consider the idempotent $u$ as a function on $\Delta(A)$ so that for any continuous function $\varphi: E \rightarrow \mathbb{C}$, the function $\widetilde{\varphi}=u \varphi$ is the trivial extension of the function $\varphi$ to the space $\Delta(A)$. Since the Banach algebras $A / I$ and $A u$ are isomorphic under the natural mapping $a+I_{u} \mapsto a u$ instead of the algebra $A / I$, we can work with the algebra $A u$. So we have the following result.

Proposition 4.5. Let $u \in A^{* *}$ be an idempotent element of $A^{* *}$ such that the space

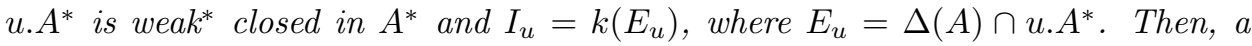
bounded continuous function $\varphi: E_{u} \rightarrow \mathbb{C}$ is a multiplier of the quotient algebra $A / I_{u}$ iff $\widetilde{\varphi} \widehat{A} \subseteq u \widehat{A}$.

We finish the paper with some questions and observations.

\section{Questions AND OBSERVATIONS}

In this final section we have collected a few questions and remarks related to the results presented in the preceding sections. In this section we suppose that $A$ is a commutative semisimple Banach algebra with a BAI.

Question 1. Let $A$ be a commutative semisimple $F$-algebra with a BAI [25]; i.e. $A^{*}$ is a $C^{*}$-algebra such that the identity element of $A^{*}$ is in the spectrum of $A$. Suppose that $X$ is a weak* closed invariant subspace of $A^{*}$ which is the range of a natural projection. Is $X$ then the range of an invariant projection? Is this the case if every maximal ideal of $A$ has a BAI? (Compare with Theorem 3.3.)

Question 2. If a closed ideal $I$ of $A$ has a BAI, then the space $I^{\perp}$ is complemented in the space $A^{*}$ so that $I^{\perp}$ is isomorphic to a quotient space of $A^{*}$. This fact raises a few questions: a) Characterize the closed ideals $I$ of $A$ such that the space $I^{\perp}$ is isomorphic to a quotient space of $A^{*}$. b) If $I$ is a closed ideal of the algebra $L^{1}(G)$ ( $G$ is abelian) and $I^{\perp}$ is isomorphic to a quotient space of $L^{\infty}(G)$, then does $I$ have a BAI? c) It is known that the Hardy space $H^{\infty}(T)$ is not complemented in $L^{\infty}(T)$ [12, p. 155]. Is it isomorphic to a quotient space of $L^{\infty}(T)$ ?

Question 3. Suppose that the complemented ideals of $A$ have BAI's. This is the case for instance for the algebra $L^{1}(G)\left(G\right.$ abelian) [1], so they are of the form $I_{u}$ for some idempotent $u \in A^{* *}$. Is it possible to characterize the idempotent elements $u$ of $A^{* *}$ for which the ideal $I_{u}$ is complemented in $A$, in particular in the case where $A=L^{1}(G)$ ?

Question 4. Let $u \in A^{* *}$ be an idempotent element of $A^{* *}$. If the space $u$. $A^{*}$ is weak ${ }^{*}$ closed in $A^{*}$, then the set $E_{u}=\{\gamma \in \Delta(A): u(\gamma)=1\}$ is closed in $\Delta(A)$ (Corollary 2.4). Conversely, if the set $E_{u}=\{\gamma \in \Delta(A): u(\gamma)=1\}$ is closed in $\Delta(A)$, is the space $u . A^{*}$ then weak ${ }^{*}$ closed in $A^{*}$ ? Is this true in the case where $A=A(G)(G$ is amenable)? 
Question 5. Theorem 4.1 shows that if $u \in A^{* *}$ is an idempotent, $A u$ is closed in $A^{* *}$ and $A^{* *}$ is WSC, then the quotient space $A / I_{u}$ is WSC. Is it enough in this result to assume, instead of $A^{* *}$, that $A$ is WSC?

Question 6. Proposition 3.4 shows that if $I$ is a closed ideal of $A$ and $I^{\perp}$ is naturally complemented in $A^{*}$, then the ideal $I$ has a $\Delta$-weak BAI. Is the converse of this result true?

Question 7. When is a natural projection $P: A^{*} \rightarrow A^{*}$ invariant? Given a natural projection $P: A^{*} \rightarrow A^{*}$ with a weak* closed range, when is there an invariant projection $Q: A^{*} \rightarrow A^{*}$ having the same range as $P$ ?

Question 8. Let $\phi: A \rightarrow A$ be a homomorphism. Then $u=\phi^{* *}(e)$ is an idempotent in $A^{* *}$. When does the ideal $I_{u}=\left\{a \in A: a \phi^{* *}(e)=0\right\}$ have a BAI? When can we realize $\operatorname{ker}(\phi)$ as $I_{v}$ for some idempotent $v \in A^{* *}$ ?

Question 9. Consider the disc algebra $A(D)$ and the ideal $I=\{a \in A(D): a(0)=$ $0\}$. This maximal ideal $I$ of $A(D)$ is complemented in $A(D)$ but does not have a BAI nor a $\Delta$-weak BAI. Let $P: A(D) \rightarrow I$ be the mapping defined by $P(a)=a-a(0) 1$. The mapping $P$ is a projection from $A(D)$ onto $I$ so that $I-P^{*}$ is a projection from $A(D)^{*}$ onto $I^{\perp}$. This projection is weak*-weak* continuous but not natural. This raises the question: If $P: A^{*} \rightarrow A^{*}$ is a weak*-weak* continuous projection, when is $P$ natural? Is this the case if every maximal ideal of $A$ has a BAI?

Question 10. Characterize those elements $m$ of $A^{* *}$ for which the space $m . A^{*}$ is weak* closed in $A^{*}$. If $A$ is an ideal in its second dual, then, for any $m \in A^{* *}$, the space $m . A^{*}$ is weak* closed in $A^{*}$ whenever it is norm closed since then the mapping $T(a)=a m$ applies $A$ into itself and $T^{*}\left(A^{*}\right)=m \cdot A^{*}$.

Question 11. For any $m \in A^{* *}$, the set $I_{m}=\{a \in A: a m=0\}$ is a closed ideal in $A$. What should $m$ be for the ideal $I_{m}$ to have a BAI?

Question 12. For any $\varphi \in A^{*}$, the set $I_{\varphi}=\{a \in A: a . \varphi=0\}$ is a closed ideal in $A$. Suppose that, for each $\gamma \in \Delta(A)$, the ideal $\operatorname{ker}(\gamma)$ has a BAI. For which kind of $\varphi \in A^{*}$ does $I_{\varphi}$ have a $\mathrm{BAI}$ ?

Question 13. Is there a $\varphi \in A^{*}$ such that the subpace $X=\left\{m \cdot \varphi: m \in A^{* *}\right\}$ of $A^{*}$ is infinite dimensional and weak* closed in $A^{*}$ ?

\section{REFERENCES}

[1] D. Alspach, A. Matheson, and J. Rosenblatt, Projections onto translation-invariant subspaces of $L_{1}(G)$, J. Funct. Anal. 59 (1984), no. 2, 254-292, DOI 10.1016/0022-1236(84)90074-0. MR.766491 (86h:43002)

[2] H. G. Dales, Banach algebras and automatic continuity, London Mathematical Society Monographs. New Series, vol. 24, The Clarendon Press Oxford University Press, New York, 2000. Oxford Science Publications. MR.1816726 (2002e:46001)

[3] A. Derighetti, Some results on the Fourier-Stieltjes algebra of a locally compact group, Comment. Math. Helv. 45 (1970), 219-228. MR0412735 (54 \#856)

[4] Pierre Eymard, L'algèbre de Fourier d'un groupe localement compact (French), Bull. Soc. Math. France 92 (1964), 181-236. MR0228628 (37 \#4208)

[5] Brian Forrest, Amenability and bounded approximate identities in ideals of $A(G)$, Illinois J. Math. 34 (1990), no. 1, 1-25. MR.1031879 (92e:43003)

[6] Brian Forrest, Projective operator spaces, almost periodicity and completely complemented ideals in the Fourier algebra, Rocky Mountain J. Math. 41 (2011), no. 1, 155-176, DOI 10.1216/RMJ-2011-41-1-155. MR2845938 
[7] Brian Forrest, Amenability and ideals in $A(G)$, J. Austral. Math. Soc. Ser. A 53 (1992), no. 2, 143-155. MR 1175708 (93i:43002)

[8] Brian E. Forrest and Volker Runde, Amenability and weak amenability of the Fourier algebra, Math. Z. 250 (2005), no. 4, 731-744, DOI 10.1007/s00209-005-0772-2. MR2180372 (2007k:43005)

[9] B. Forrest, E. Kaniuth, A. T. Lau, and N. Spronk, Ideals with bounded approximate identities in Fourier algebras, J. Funct. Anal. 203 (2003), no. 1, 286-304, DOI 10.1016/S00221236(02)00121-0. MR1996874(2004e:43002)

[10] A. Ya. Helemskii, The homology of Banach and topological algebras, Mathematics and its Applications (Soviet Series), vol. 41, Kluwer Academic Publishers Group, Dordrecht, 1989. Translated from the Russian by Alan West. MR 1093462 (92d:46178)

[11] Edwin Hewitt and Kenneth A. Ross, Abstract harmonic analysis. Vol. II: Structure and analysis for compact groups. Analysis on locally compact Abelian groups, Die Grundlehren der mathematischen Wissenschaften, Band 152, Springer-Verlag, New York, 1970. MR0262773 (41 \#7378)

[12] Kenneth Hoffman, Banach spaces of analytic functions, Prentice-Hall Series in Modern Analysis, Prentice-Hall Inc., Englewood Cliffs, N. J., 1962. MR0133008 (24 \#A2844)

[13] B. Host, Le théorème des idempotents dans $B(G)$ (French, with English summary), Bull. Soc. Math. France 114 (1986), no. 2, 215-223. MR860817 (88b:43003)

[14] John E. Gilbert, On projections of $L^{\infty}(G)$ onto translation-invariant subspaces, Proc. London Math. Soc. (3) 19 (1969), 69-88. MR0244705 (39 \#6019)

[15] I. Glicksberg and I. Wik, Multipliers of quotients of $L_{1}$, Pacific J. Math 38 (1971), 619-624. MR0306813(46 \#5935)

[16] Gilles Godefroy, On Riesz subsets of abelian discrete groups, Israel J. Math. 61 (1988), no. 3, 301-331, DOI 10.1007/BF02772575. MR941245 (89m:43011)

[17] Gilles Godefroy, Sous-espaces bien disposés de $L^{1}$-applications (French, with English summary), Trans. Amer. Math. Soc. 286 (1984), no. 1, 227-249, DOI 10.2307/1999403. MR756037 (86h:46033)

[18] Frederick P. Greenleaf, Invariant means on topological groups and their applications, Van Nostrand Mathematical Studies, No. 16, Van Nostrand Reinhold Co., New York, 1969. MR0251549(40 \#4776)

[19] Jyunji Inoue and Sin-Ei Takahasi, Constructions of bounded weak approximate identities for Segal algebras on LCA groups, Acta Sci. Math. (Szeged) 66 (2000), no. 1-2, 257-271. MR 1768865 (2001i:43004)

[20] Charles A. Jones and Charles D. Lahr, Weak and norm approximate identities are different, Pacific J. Math. 72 (1977), no. 1, 99-104. MR0447972 (56 \#6282)

[21] Eberhard Kaniuth, A course in commutative Banach algebras, Graduate Texts in Mathematics, vol. 246, Springer, New York, 2009. MR2458901(2010d:46064)

[22] E. Kaniuth, A. T. Lau, and A. Ülger, Multipliers of commutative Banach algebras, power boundedness and Fourier-Stieltjes algebras, J. Lond. Math. Soc. (2) 81 (2010), no. 1, 255-275, DOI 10.1112/jlms/jdp068. MR2580464(2011c:46107)

[23] Andrew G. Kepert, The range of group algebra homomorphisms, Canad. Math. Bull. 40 (1997), no. 2, 183-192, DOI 10.4153/CMB-1997-022-6. MR 1451272 (98m:43005)

[24] Anthony To Ming Lau, Invariantly complemented subspaces of $L_{\infty}(G)$ and amenable locally compact groups, Illinois J. Math. 26 (1982), no. 2, 226-235. MR650390 (83g:43002)

[25] Anthony To Ming Lau, Analysis on a class of Banach algebras with applications to harmonic analysis on locally compact groups and semigroups, Fund. Math. 118 (1983), no. 3, 161-175. MR736276 (85k:43007)

[26] Anthony To Ming Lau and Viktor Losert, Weak*-closed complemented invariant subspaces of $L_{\infty}(G)$ and amenable locally compact groups, Pacific J. Math. 123 (1986), no. 1, 149-159. MR834144 (87g:43001)

[27] Ronald Larsen, An introduction to the theory of multipliers, Springer-Verlag, New York, 1971. Die Grundlehren der mathematischen Wissenschaften, Band 175. MR0435738 (55 \#8695)

[28] Horst Leptin, Sur l'algèbre de Fourier d'un groupe localement compact (French), C. R. Acad. Sci. Paris Sér. A-B 266 (1968), A1180-A1182. MR0239002 (39 \#362)

[29] Gerald M. Leibowitz, Lectures on complex function algebras, Scott, Foresman and Co., Glenview, Ill., 1970. MR0428042 (55 \#1072) 
[30] Teng-Sun Liu, Arnoud van Rooij, and Ju Kwei Wang, Projections and approximate identities for ideals in group algebras, Trans. Amer. Math. Soc. 175 (1973), 469-482. MR0318781 (47 \#7327)

[31] Alan L. T. Paterson, Amenability, Mathematical Surveys and Monographs, vol. 29, American Mathematical Society, Providence, RI, 1988. MR961261 (90e:43001)

[32] Haskell P. Rosenthal, Projections onto translation-invariant subspaces of $L^{p}(G)$, Mem. Amer. Math. Soc. No. 63 (1966), 84. MR0211198 (35 \#2080)

[33] P. F. Renaud, Invariant means on a class of von Neumann algebras, Trans. Amer. Math. Soc. 170 (1972), 285-291. MR.0304553 (46 \#3688)

[34] Walter Rudin, Projections on invariant subspaces, Proc. Amer. Math. Soc. 13 (1962), 429432. MR0138012(25 \#1460)

[35] Walter Rudin, Functional analysis, 2nd ed., International Series in Pure and Applied Mathematics, McGraw-Hill Inc., New York, 1991. MR1157815 (92k:46001)

[36] Walter Rudin, Fourier analysis on groups, Wiley Classics Library, John Wiley \& Sons Inc., New York, 1990. Reprint of the 1962 original; a Wiley-Interscience Publication. MR,1038803 (91b:43002)

[37] Volker Runde, Lectures on amenability, Lecture Notes in Mathematics, vol. 1774, SpringerVerlag, Berlin, 2002. MR 1874893 (2003h:46001)

[38] Bert M. Schreiber, On the coset ring and strong Ditkin sets, Pacific J. Math. 32 (1970), 805-812. MR0259502 (41 \#4140)

[39] Peter J. Wood, Complemented ideals in the Fourier algebra of a locally compact group, Proc. Amer. Math. Soc. 128 (2000), no. 2, 445-451, DOI 10.1090/S0002-9939-99-04989-8. MR.1616589 (2000c:43004)

Department of Mathematical and Statistical Sciences, University of Alberta, Edmonton, Alberta, Canada T6G 2G1

E-mail address: tlau@math.ualberta.ca

Department of Mathematics, Koc University, 34450 Sariyer, Istanbul, Turkey

E-mail address: aulger@ku.edu.tr 